\title{
Ageing and autism: A 4-year follow-up study of mental health and quality of life in autistic adults
}

\author{
Amanda Roestorf, $\mathrm{PhD}^{1}$, Patricia Howlin ${ }^{1}$, and Dermot M. Bowler ${ }^{1}$ \\ ${ }^{1}$ Affiliation not available
}

June 30, 2022

\begin{abstract}
Background: Poor mental health is known to adversely affect functional abilities, social isolation and quality of life (QoL). It is, therefore, crucial to consider the long-term impacts of mental health conditions as autistic adults grow older. Objectives: To explore, in a group of community-based autistic adults, the extent of: (i) autistic traits, co-occurring physical and mental health conditions; (ii) age-related differences in those conditions, and changes over time; and (iii) their impact on everyday living and QoL. Method: 68 autistic adults (aged 19-80 years) participated in the first study (T1); 49 participants from T1 took part in a follow-up at T2 (mean retest interval 2.4 years). Standardised self-report measures of autistic traits, mental health and QoL were completed at both time points. Results: Over two-thirds (71\%) of autistic adult participants experienced at least one co-occurring condition, and over a third (37\%) met the criteria for three or more co-occurring conditions. Mental and physical health difficulties were related to autistic traits and difficulties in everyday life and were consistent predictors of poor QoL at T1 and T2. Conclusion: Mental health difficulties in autism persisted into older age and did not improve over time. These findings have important implications for mental health provision for autistic adults in older age.

Pre-print article
\end{abstract}

